\title{
Instrumentation Amplifier for the Heart Rate Measurement with Low Power Consumption
}

\author{
Faezeh Zeinalzadeh ${ }^{1}$, Maryam Zare ${ }^{2}$ \\ ${ }^{1}$ MSc student of Imam Reza International University \\ ${ }^{2}$ Assistant professor of Imam Reza International University
}

\begin{abstract}
Medical Instrumentation amplifiers are one of the most important parts in biologic signal recording systems which have various application in medicine. The main challenges in these circuits designing come from the nature of biologic signals. The amplifying circuit should work in low power to prevent the tissues heating and doesn't disturb the tissue cells. In this paper an amplifier in CMOS $0.18 \mu \mathrm{m}$ has been designed for the power consumption reduction and prevention of patient skin disturbing that only uses $7 \mu w$. Also the amplifier should use low level of silicon to reduce the amount of implantable medical sensor. For the chip size reduction in designing the MOSFET transistors has been utilized instead of passive resistances.
\end{abstract}

Keywords: Electrocardiogram, Analog Integrated Circuit, Biological signal amplifier, heart signal measurement, Medical amplifier with low power consumption

\section{Introduction}

Electrocardiogram is a vehicle which received heart electrical currents via some attached electrodes on different parts of skin and plots them as a graph. This graph is called Electrocardiogram. It has been remained as one of the most important devices in medical determinable tools since 1901. The medical devices at first received the low frequency biological signals. These signals should be amplified because of their low frequency [1]. Then the analog signal amplified and convert to the digital ones. Finally the digital signals will be processed. Biological signal amplifier must have low input noise to allow the low frequency biologic signals, many $10 \mu$ volt, determined. Extra information could be found in Harrison [2] (2003). In presented structure in this article the middle band gain reaches to $40 \mathrm{~dB}$, it has been designed in $1.5 \mu \mathrm{m}$ CMOS technology. Its bandwidth is $0.025 \mathrm{~Hz}$ to $7.2 \mathrm{KHz}$ and has $80 \mu \mathrm{w}$ consumption supplies. After that in 2007 [3] and 2011 [4] some different amplifiers were designed by Lim et al and Majidzadeh et al respectively. In second part of this article the challenges against of medical amplifiers will be introduced and after that a medical amplifier with low supply and noise. In part four two-stage amplifier and its particles will be explained. Finally the results of similar works will be compared in consumption supply reduction domain.

\section{Medical Amplifier Designing Challenges}

There are challenges in medical amplifier designing because of biological signals individual properties. Heart signal domain is in 0.5-4 mv range which should be amplified before the processing [5]. So we need an amplifier with high difference gain. The amplifier must be programmable because of difference in signal receive location and differences from a person to the other. It should have the gain range from 100 to 1000 [5]. In this situation it provides enough amplifying power equal to $100 \mu \mathrm{v}$ to $10 \mathrm{mv}$.

In amplifier designing, the noise reduction is one of the most important factors. The smallest frequency in heart signal is wave p. it has 200-300 $\mu \mathrm{v}$ domain. The input noise should be lower than $200 \mu \mathrm{v}$ to let know the heart signal [6]. The DC offset is generated because of skin electrode interference. This offset is about $300 \mathrm{mv}$ that generally is many times bigger that measured signal voltage. In lack of enough damping, the offset fills the input stage. Needed bandwidth for heart signal measurement depends on its application. For non-determination applications the 0.5 to $40 \mathrm{~Hz}$ is popular and for determination applications it should be 0.05 to $150 \mathrm{~Hz}$.

\section{Medical Amplifier With Low Supply Consumption And Noise Number}

Harrison study [2] is one of the most basic works in medical amplifier designing with low noise and supply consumption. In presented structure middle band gain from the $C_{1} / C_{2}$ relation reaches to $40 \mathrm{~dB}$, in figure $1 \mathrm{C} 1$ and $\mathrm{C} 2$ are the capacitor feedback network and $\mathrm{M}_{\mathrm{a}}$ to $\mathrm{M}_{\mathrm{d}}$ transistors have been utilized as the semiresistors. These resistors are biased in under threshold area to have the high resistivity. This structure has been designed in $1.5 \mu \mathrm{m}$ CMOS. Its bandwidth is equal to $0.025 \mathrm{~Hz}$ to $7.2 \mathrm{KHz}$ and its consumption supply is equal to $80 \mu \mathrm{w}$. this structure has been considered as the base of many works. 


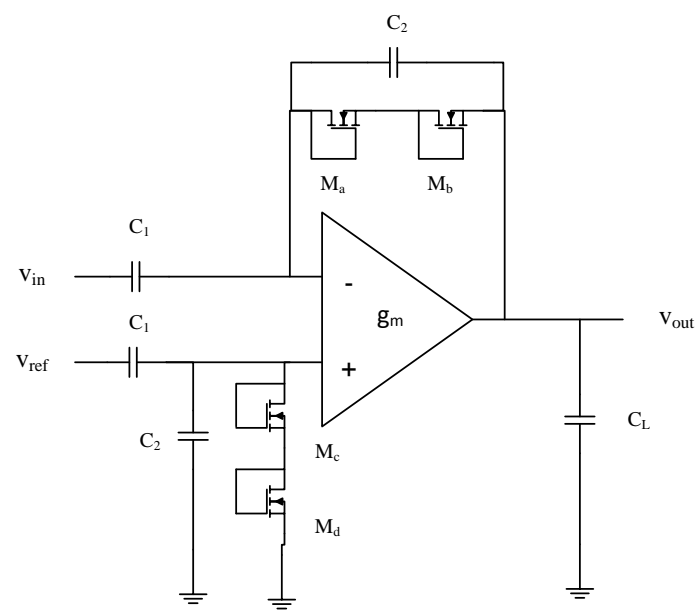

Fig1. Schematic of Neural amplifier [2]

\section{Two-Stage Amplifier Designing In Low Consumption Supply}

Amplifier structure has the transistor semi-resistor for amplifying of the low frequency signals in $\mathrm{MHz}$ range and eliminates the DC offset simultaneously. It has 2 stage and feeds from the DC voltage. First stage is a differential structure that supplies the high gain. Second stage has been designed for the suitable suing achievement.

For the flicker noise reduction, the $\mathrm{p}$ transistors have been utilized as the input fragments. Because the noise of $p$ transistors is lower than type $n$. amplifier suing of open loop is 0.5 volt. $R_{c}$ and $C_{c}$ caused the stability of the circuit. Gained current of feeding source has been considered equal to $3 \mu \mathrm{A}$. the transistor dimensions have been calculated based on current amount. The gain of open loop structure reaches to $48 \mathrm{~dB}$ in this amplifier.

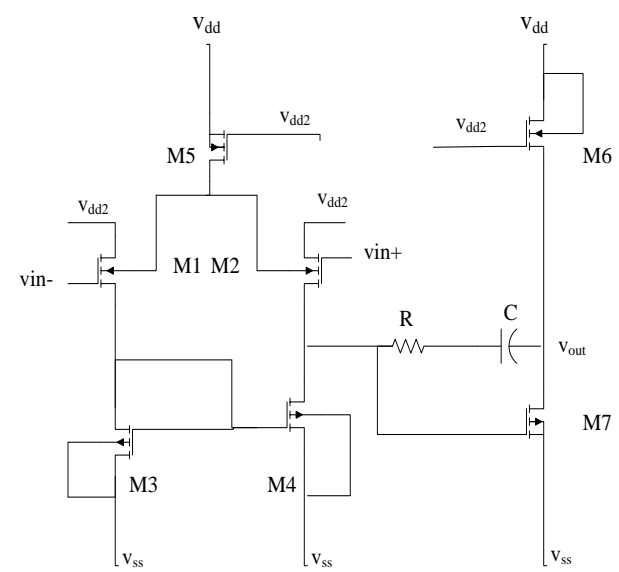

Fig2. Schematic of two-stage OTA [7]

\section{Close Loop Structure}

The closed loop structure is same as figure 1. In this structure the $M_{a}$ to $M_{d}$ transistors have been utilized as the semi-resistors. The closed loop gain is created by the $c_{1}$ and $c_{2}$ capacitor feedback. If the gain of opened loop up amp be greater than $\mathrm{C}_{1} / \mathrm{C}_{2}$, the middle band gain of closed loop up amp $\left(\mathrm{A}_{\mathrm{m}}\right)$ will be exactly equal to $\mathrm{C}_{1} / \mathrm{C}_{2}$. In gain calculation, the impedance consideration passed up. Low frequency of amplifier will almost be equals to relation 1 .

$$
f_{l}=\frac{1}{2 \pi \cdot R \cdot C_{2}}
$$

Low level frequency of this circuit is equal to $6 \mathrm{Htz}$. High level frequency is also equals to relation 2.

$$
f_{H}=\frac{G_{m}}{2 \pi \cdot A_{m} \cdot C_{l}}
$$

For the maximum gain achievement of amplifier in single stage structure, the c2 should be select 
minimum and $\mathrm{c} 1$ should be enormous. Based on measured properties in reference paper [2], this amplifier has the $7.7 \mathrm{KHz}$ bandwidth. This structure level frequency is adjustable via the $\mathrm{C}_{\mathrm{L}}$ capacitor changing and loop gain reduction. $\quad v_{r e f}=\frac{v_{d d}}{4}$ Feeding, is located for the achieving of reasonable gain and filling the output stage transistors.

$\mathrm{V}_{\text {int }}$ input is similar to heart signal with $4 \mathrm{mv}_{\mathrm{p}-\mathrm{p}}$ domain and frequency equal to $100 \mathrm{~Hz}$. Reducing the domain to $1 \mathrm{mv}_{\mathrm{p}-\mathrm{p}}$ the results don't change and is stable towards the domain changing. Limiting the current to consumption supply to $7 \mu \mathrm{w}$, the closed loop gain equal to $40 \mathrm{~dB}$, ECG is the difference measurement between two electrodes. Because the domain of biological signals is about mv or lower than it. CMRR is the circuit difference gain on its meant gain mode. PSRR of a circuit is the circuit difference gain on amplified feeding source noise. For the feed source error calculation, a sinusoidal signal equal to 10 percent of DC voltage of feeding source, has been added to the feeding source. In this structure the amount of CMRR and PSRR have been obtained 57.8 and $48.8 \mathrm{~dB}$ respectively.

\section{Semi-Resistor Elements}

The $\mathrm{M}_{\mathrm{a}}$ to $\mathrm{M}_{\mathrm{d}}$ transistors has been considered as the semi-resistor. Each transistor semi-resistor has the $0.48 \mu \mathrm{m} \times 0.48 \mu \mathrm{m}$ size. For the low voltages cross the device this rising resistivity is very much. For $|\Delta \mathrm{V}|<0.2$ $\mathrm{v}$, the $\mathrm{v}$ changes towards i has been measured as relation 3:

$$
\frac{d v}{d i}>10^{11} \Omega
$$

The exact measurement of $\frac{d v}{d i}$ is so difficult because of the low current. Two bipolar transistors are used for the output signal distortion reduction. The low level frequency $\mathrm{W}_{\mathrm{L}}$ amplifier with $\mathrm{AC}$ coupling is equal to $\frac{1}{2 r_{i n c} \times C_{2}}$. Considering Long time constant, the huge changes at input caused the enormous voltage on bipolar offset, increasing resistivity reduced but the subsidence time will be more rapid.

\section{Measurement Noise Efficiency Factor}

The relation of power and noise should be known for the noise minimizing. NEF explains this relation quantitatively. $V_{\text {in }}$ is the effective input noise voltage. $I_{\text {total }}$ is the amplifier whole current and $\mathrm{BW}$ is the bandwidth in Hz. NEF number for bipolar transistor is an amplifier without noise is equal to 1. In this structure the input noise is $1.2 \mu \mathrm{v}_{\mathrm{rms}}$.

$$
N E F=V_{n i, r m s} \sqrt{\frac{2 I_{\text {total }}}{\left(\Pi \cdot U_{T} \cdot 4 K T \cdot B W\right)}}
$$

\section{Conclusion}

In this article the challenges against of circuit designing, was assessed. After that the heart signal amplifier fragments explained. These kind of circuits work on high range of power, which warms the patient skin surface and hurts its tissue. In this design it has been tried to prevent the problem via the consumption supply reduction to $7 \mu \mathrm{w}$. also it provides the consumption supply in biological signal amplifier. Table 1 shows the results comparison.

Table 1. Results comparison

\begin{tabular}{|l|l|l|l|}
\hline Parameter & {$[2]$} & {$[4]$} & This Work \\
\hline Technology & $1.5 \mu \mathrm{m}$ & $0.18 \mu \mathrm{m}$ & $0.18 \mu \mathrm{m}$ \\
\hline Supply Voltage(v) & \pm 2.5 & 1.8 & \pm 1 \\
\hline Supply current $(\mu \mathrm{A})$ & 16 & N/A & 3 \\
\hline Gain $(\mathrm{dB})$ & 39.5 & 39.4 & 40 \\
\hline Bandwidth(KHZ) & 7.2 & 7.2 & 7.7 \\
\hline Low Frequency(HZ) & 0.025 & N/A & 0.78 \\
\hline $\begin{array}{l}\text { Input-referred } \\
\text { noise }\left(\mu \mathrm{V}_{\text {rms }}\right)\end{array}$ & 2.2 & 3.5 & 1.2 \\
\hline CMRR $(\mathrm{dB})$ & 83 & 70.1 & 57.8 \\
\hline PSRR $(\mathrm{dB})$ & 85 & 63.8 & 48.9 \\
\hline
\end{tabular}




\section{References}

[1]. Rezaee-Dehsorkh, H.; Ravanshad, N.; Lotfi, R.; Mafinezhad, K.; Sodagar, A M., "Analysis and Design of Tunable Amplifiers for Implantable Neural Recording Applications," Emerging and Selected Topics in Circuits and Systems, IEEE Journal on , vol.1, no.4, pp.546,556, Dec. 2011

[2]. Harrison, R.R.; Charles, C., "A low-power low-noise CMOS amplifier for neural recording applications," Solid-State Circuits, IEEE Journal of , vol.38, no.6, pp.958,965, June 2003.

[3]. Lim, E.C.M.; Xiaodan Zou; Yuanjin Zheng; Jun Tan, "Design of Low-Power Low-Voltage Biomedical Amplifier for Electrocardiogram Signal Recording," Biomedical Circuits and Systems Conference, 2007. BIOCAS 2007. IEEE , vol., no., pp.191,194, 27-30 Nov. 2007.

[4]. Majidzadeh, V.; Schmid, A; Leblebici, Y., "Energy Efficient Low-Noise Neural Recording Amplifier With Enhanced Noise Efficiency Factor," Biomedical Circuits and Systems, IEEE Transactions on, vol.5, no.3, pp.262,271, June 2011.

[5]. John G. Webster, Medical Instrumentation: Application and Design,John Wiley \& Sons, 1998.

[6]. Margaret (Maggie) K. Delano, "A Long Term Wearable Electrocardiogram (ECG) Measurement System" , Master thesis, 2012.

[7]. Palmisano,G.; Palumbo,G.; Pennisi,S.,"Design procedure for two-stage cmos transconductance operational amplifiers: a tutorial," Analog Integrated Circuits And Signal Processing, vol.27, no.3, pp.179,189, June. 2001. 